\title{
Severe Multiple Sclerosis Manifesting upon GnRH Agonist Therapy for Uterine Fibroids
}

\author{
Kenzo Sakurai ${ }^{1}$, Kensuke Shinohara ${ }^{1}$, Takeshi Imai ${ }^{1}$, \\ Yoshihisa Yamano $^{2}$ and Yasuhiro Hasegawa ${ }^{2}$
}

\begin{abstract}
:
This case concerned a 39-year-old woman diagnosed with uterine fibroids. Upon initiation of gonadotropin-releasing hormone $(\mathrm{GnRH})$ agonist therapy, she experienced various neurological deficits but did not seek medical attention because of gradual spontaneous symptom improvement. Upon completing four courses of GnRH agonist therapy, she began experiencing severe neurological symptoms and was diagnosed with multiple sclerosis (MS). Although her symptoms improved with steroid pulse therapy, serious sequelae remained. GnRH agonist therapy can exacerbate the disease activity of MS; therefore, awareness of the potential emergence of neurological symptoms during GnRH agonist therapy is important.
\end{abstract}

Key words: assisted reproductive technology, estrogen, GnRH agonist therapy, multiple sclerosis, uterine fibroids

(Intern Med 59: 3093-3096, 2020)

(DOI: 10.2169/internalmedicine.4839-20)

\section{Introduction}

Multiple sclerosis (MS) is an inflammatory demyelinating disease commonly seen in young women in their 20s and 30s (1). Globally, an estimated 2.3 million people are affected by MS. The number of patients with MS in Japan has rapidly increased (2), with a current estimate of 13,000 people affected.

Gonadotropin-releasing hormone $(\mathrm{GnRH})$ agonist therapy is widely employed for the treatment of estrogen-dependent diseases such as uterine fibroids and endometriosis. In Japan, a buserelin acetate nasal solution was approved as the first GnRH agonist preparation in 1988; today, four different GnRH agonists available in five dosage forms are being used. GnRH agonists are effective against uterine fibroids in terms of reducing the fibroid size and uterine blood flow, which helps minimize intraoperative blood loss (3). Therefore, combined with surgical treatment, GnRH agonist therapy is an effective treatment option. However, estrogen suppression by $\mathrm{GnRH}$ agonists may induce a decrease in bone mineral content, menopause-like symptoms, and atypical genital bleeding. Furthermore, one case of MS recurrence affected by GnRH agonist therapy has been described (4). To our knowledge, however, there are no reports of patients in whom a diagnosis of MS was established only in the wake of GnRH agonist therapy.

We herein report a case of MS in which physical symptoms were sufficiently clear to diagnose MS only after being triggered by $\mathrm{GnRH}$ agonist therapy administered to treat uterine fibroids.

\section{Case Report}

- Patient: A 39-year-old woman.

- Chief complaint: Gait impairment, numbness in the left upper limb, dysuria.

- Medical history: Uterine fibroids, sinusitis.

- Family history: No special notes.

- Personal history: The patient was born in the Philippines and had lived in Japan for more than 20 years.

- History of present illness: The patient had been diagnosed with uterine fibroids in April 2018. Despite recommendations, the patient did not wish to undergo surgery be-

${ }^{1}$ Department of Neurology, Kawasaki Municipal Tama Hospital, Japan and ${ }^{2}$ Department of Neurology, St. Marianna University School of Medicine, Japan

Received: March 12, 2020; Accepted: June 22, 2020; Advance Publication by J-STAGE: August 4, 2020

Correspondence to Dr. Kenzo Sakurai, k2sakurai@marianna-u.ac.jp 
Table. Laboratory Findings on Admission.

\begin{tabular}{lclc}
\hline Serology and immunology & \multicolumn{3}{l}{ Cerebrospinal fluid examination } \\
Antinuclear antibody & $<40$ times & protein & $48.9 \mathrm{mg} / \mathrm{dL}$ \\
Anti ds-DNA antibody & $2.5 \mathrm{IU} / \mathrm{mL}$ & cell & 21 \\
Anti SS-A antibody & $2.8 \mathrm{U} / \mathrm{mL}$ & multi & $0 \%$ \\
Anti SS-B antibody & $2.9 \mathrm{U} / \mathrm{mL}$ & mono & $100 \%$ \\
Anti cardiopyrpin conjugate antibody & $<8.0 \mathrm{U} / \mathrm{mL}$ & glucose & $64 \mathrm{mg} / \mathrm{dL}$ \\
Anti TPO antibody & $<0.50 \mathrm{IU} / \mathrm{mL}$ & Oligoclonal band positive & \\
Anti thyroglobulin antibody & $1.18 \mathrm{IU} / \mathrm{mL}$ & Myelin basic protein & $889 \mathrm{mg} / \mathrm{dL}$ \\
Anti aquaporin-4 & $<1.5 \mathrm{U} / \mathrm{mL}$ & IgG index & 0.64 \\
Anti HTLV-1 antibody & - & & - \\
MPO-ANCA & - & Female hormone & $6.72 \mathrm{mIU} / \mathrm{mL}$ \\
PR3-ANCA & - & FSH & $0.77 \mathrm{mIU} / \mathrm{mL}$ \\
IgG & $1,747 \mathrm{mg} / \mathrm{dL}$ & LH & $<10 \mathrm{pg} / \mathrm{dL}$ \\
IgM & $262 \mathrm{mg} / \mathrm{dL}$ & E2 & \\
IgA & $190 \mathrm{mg} / \mathrm{dL}$ & & \\
ACE & $14.8 \mathrm{IU} / \mathrm{L}$ & & \\
IL-2R & $69 \mathrm{U} / \mathrm{mL}$ & & \\
\hline
\end{tabular}

ACE: angiotensin converting enzyme, IL-2R: interleukin-2 receptor, TPO: thyroid peroxidase

cause she had no children, so she was followed up for a while. However, since she experienced frequent atypical bleeding, GnRH agonist therapy (leuprorelin acetate; a subcutaneous injection of $30 \mu \mathrm{g} / \mathrm{kg}$ given every 4 weeks) was initiated. After the second course of therapy was completed in January 2019, the patient experienced paresthesia of the right lower limb, followed by paresthesia of the left lower limb, gait impairment, and dysuria. However, this was not subject to further investigation because of gradual symptom relief. She again became aware of weakness in the right lower limb in March, blurriness in her eyes in April, and weakness that developed in her left lower limb in August, but again, her symptoms gradually diminished. After the patient had received the fourth course of GnRH agonist therapy in October, the patient was hospitalized in our hospital in November for recurrence of gait impairment, numbness in the left upper limb, and dysuria.

- Neurological findings: Lucid consciousness with no disturbance of orientation. No abnormalities were observed in the cranial nerves. Manual muscle testing disclosed right dominance and a muscle decrease in the lower limbs as follows: iliopsoas muscle, 4/4; anterior tibial muscle and pollicis longus muscle, 4/5. Paresthesia was noted in the left side of the body below the cervical region as well as in the right lower limb. The distal part of the left knee joint exhibited a reduced sense of vibration. The knee-heel test revealed bilaterally hypermetria. The deep tendon reflex was increased in all four limbs. The Babinski reflex test was present bilaterally. The patient found it difficult to stand up. Mild bladder and rectal disturbances were noted.

- Laboratory findings: CRP $<0.01 \mathrm{mg} / \mathrm{dL}$, estradiol $<10$ $\mathrm{pg} / \mathrm{mL}$. Antinuclear antibody, anti-SS-A antibody, anti-SS-B antibody, anti-TPO antibody, and anti-aquaporin 4 (AQP4) antibody (enzyme-linked immunosorbent assay) were all negative (Table). Cerebrospinal fluid test results were as follows: cell count, 21; protein, $48.9 \mathrm{mg} / \mathrm{dL}$; oligoclonal band- positive; and $\mathrm{IgG}$ index, 0.64. Head magnetic resonance imaging (MRI) revealed an ovoid lesion around the lateral ventricle on T2-weighted images; T1 black hole and open ring signs were observed on contrast imaging. Spinal MRI showed several high signals on T2-weighted images, and contrast effects were observed at C7 and Th4-Th5 levels. Pelvic MRI showed a $10-\mathrm{cm}$ mass lesion with iso- to highintensity areas within the anterior wall muscle layers of the uterine body on T2-weighted images (Figure).

- Posthospitalization course: After the dissemination in space and time were proven, the positive oligoclonal band and negative AQP4 antibody test results were obtained, and other diseases ruled out. The patient was then diagnosed with relapsing-remitting MS with a severity score of 7.0 according to the Kurtzke Expanded Disability Status Scale (EDSS). No impaired vision or fundus findings were noted on an ophthalmologic examination; however, optical coherence tomography revealed thinning of the ganglion cell layers in both eyes, suggesting that the patient had developed optic neuritis previously. GnRH agonist therapy was discontinued, and steroid pulse therapy (methylprednisolone 1,000 $\mathrm{mg}$, administered intravenously for 3 days) was initiated immediately thereafter. The bladder and rectal disturbances improved, and muscular strength and paresthesia in the lower limbs also slightly improved. Upon completion of the third treatment course, the patient's daily life activities improved enough that she was able to walk with a cane, with only slight paresthesia remaining in both lower limbs. On the 29 th day, her EDSS score decreased to 4.0, and she was discharged from the hospital.

\section{Discussion}

GnRH agonist therapy increases the disease activity of MS $(4,5)$, as observed in the present case. Correale et al. reported that the risk of MS aggravation and imaging-based 

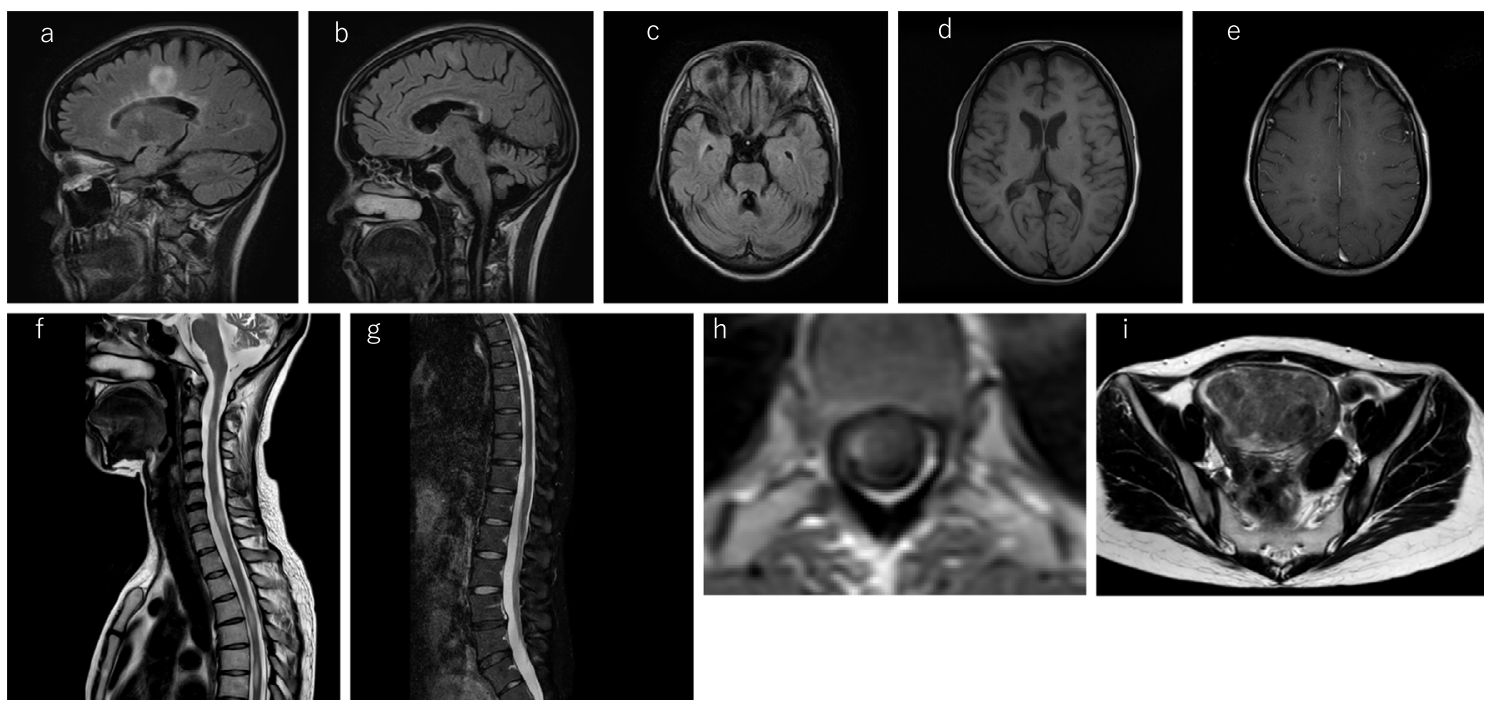

Figure. MRI findings. a: ovoid lesion (FLAIR), b: septal-callosal interface lesion (FLAIR), c: lesion of the pons (FLAIR), d: T1 black hole (T1), e: open ring sign (T1 Gd+), f/g: spinal lesion (T2), h: spinal lesion (T1 Gd+), l: uterine fibroid (T2). FLAIR: fluid-attenuated inversion recovery

recurrence increased by seven- and nine-fold, respectively (6), in MS patients who underwent GnRH agonist therapy and ovarian stimulation hormone administration for assisted reproductive technology (ART). In Japan, ARTrelated aggravation of MS is reportedly associated with the presence of recurrence in the year before MS treatment and unsuccessful fertility treatment (7). However, the present one is the first reported MS case that was diagnosed only in the wake of GnRH agonist therapy.

The likely underlying mechanism involves estrogen concentrations. High estrogen concentrations have been reported to transform Th1 to Th2 cells, reduce the number of Th17 cells, and activate Treg cells $(8,9)$. An imbalance among certain types of $\mathrm{T}$ cells, including $\mathrm{Th} 1$ cells producing proinflammatory cytokines, such as interleukin (IL)-2, interferon (IFN)- $\gamma$, and tumor necrosis factor (TNF)- $\alpha$; Th17 cells producing IL-17; Th2 cells producing IL-4, IL-5, and IL-10; and Treg cells (10) is involved in MS recurrence. Furthermore, oral contraceptives containing estrogen as the active ingredient reportedly reduce the recurrence rate of MS by $40 \%$ (11). GnRH agonists reduce the secretion of luteinizing and follicle-stimulating hormones and decrease estradiol by decreasing GnRH receptor sensitivity. GnRH agonist therapy can reduce the size of uterine fibroids, as it decreases the estrogen concentration (12), and was indeed used for this purpose in the present case. Consequently, her estradiol level on admission was remarkably low, suggesting MS activation.

As of February 2020, six different disease-modifying drugs (DMDs) are available for MS treatment in Japan. The concomitant use of a DMD has been reported to reduce the recurrence rate in patients with a definite MS diagnosis who require GnRH agonist therapy (13). Because our patient tested positive for the JC Virus index, which would necessitate treatment for non-DMD-intervened uterine fibroids, de- escalation therapy was our preferred treatment strategy. In this strategy, natalizumab (NTZ), which is a potent drug and induces an early response (within two months) $(14,15)$, is used initially; GnRH agonist therapy is then performed two months after NTZ to ensure the NTZ response, and finally, dimethyl fumarate or a similar drug is administered at least four months after the completion of the GnRH agonist therapy to minimize the risk of the emergence of NTZ antibodies (16).

The present study has two limitations. First, the patient might have had subclinical MS before GnRH agonist therapy. Therefore, while GnRH agonist therapy undoubtedly exacerbated MS, determining whether or not the therapy caused, triggered, or merely exacerbated MS is difficult. Second, contrary to this case, there is a report in which GnRH agonist therapy improved autoimmune encephalitis (17); therefore, this information should be borne in mind when further analyzing the relationship between GnRH agonist therapy and MS.

While neurologists know that pregnancy and delivery affect MS activity, our experience with the present case highlights the importance of educating physicians performing GnRH agonist therapy about its possible effects on MS.

The authors state that they have no Conflict of Interest (COI).

\section{References}

1. Goodin DS. The epidemiology of multiple sclerosis: insights to disease pathogenesis. Handb Clin Neurol 122: 231-266, 2014.

2. Osoegawa M, Kira J, Fukazawa T, et al. Temporal changes and geographical differences in multiple sclerosis phenotypes in Japanese: nationwide survey results over 30 years. Mult Scler 15: 159173, 2009.

3. Reinsch RC, Murphy AA, Morales AJ, et al. The effect of RU 486 and leuprolide acetate on uterine artery blood flow in the fibroid 
uterus: a prospective, randomized study. Am J Obstet Gynecol 170: 1623-1628, 1994.

4. Michel L, Foucher Y, Vukusic S, et al.; Club Francophone de la Sclérose En Plaques (CFSEP). Increased risk of multiple sclerosis relapse after in vitro fertilization. J Neurol Neurosurg Psychiatry 83: 796-802, 2012.

5. Torkildson $\varnothing$, Holmøy T, Myhr KM. Severe multiple sclerosis reactivation after gonadotropin treatment. Mult Scler Relat Disord 22: 38-40, 2018.

6. Correale J, Farez MF, Ysrraelit MC. Increase in multiple sclerosis activity after assisted reproduction technology. Ann Neurol 72: 682-694, 2012.

7. Simizu Y, Ikeguchi R, Kitagawa K. Pregnancy outcome, changes in lymphocyte subsets in peripheral blood, and plasma osteopontin in Japanese patients with multiple sclerosis after assisted reproductive technology (P4.369). Neurology 90 (Suppl): P4.369, 2018.

8. Bove R, Chitnis T. Sexual disparities in the incidence and course of MS. Clin Immunol 149: 201-210, 2013.

9. Savettieri G, Messina D, Andreoli V, et al. Gender-related effect of clinical and genetic variables on the cognitive impairment in multiple sclerosis. J Neurol 251: 1208-1214, 2001.

10. Hemmer B, Kerschensteiner M, Korn T. Role of the innate and adaptive immune responses in the course of multiple sclerosis. Lancet Neurol 14: 406-419, 2015.

11. Alonso A, Jick SS, Olek MJ, Ascherio A, Jick H, Hernán MA. Recent use of oral contraceptives and the risk of multiple sclerosis. Arch Neurol 62: 1362-1365, 2005.
12. Badaru A, Wilson DM, Bachrach LK, et al. Sequential comparisons of one-month and three-month depot leuprolide regimens in central precocious puberty. J Clin Endocrinol Metab 91: 18621867, 2006.

13. Brzosko B, Thiel S, Gold R, Hellwig K. Low relapse risk under disease modifying treatment during ART in women with relapsing remitting multiple sclerosis (P4. 356). Neurol 90 (Suppl): P4.356, 2018.

14. Polman $\mathrm{CH}$, O'Connor PW, Havrdova E, et al. A randomized, placebo-controlled trial of natalizumab for relapsing multiple sclerosis. N Engl J Med 354: 899-910, 2006.

15. Saida T, Kira J, et al. Efficacy, safety, and pharmacokinetics of natalizumab in Japanese multiple sclerosis patients: a double-blind, randomized controlled trial and open-labl pharmacokinetic study. Mult Scler Relat Disord 11: 25-31, 2017.

16. Oliver B, Fernández $\mathrm{O}$, Orpez $\mathrm{T}$, et al. Kinetics and incidence of antii-natalizumab antibodies in multiple sclerosis patients on treatment for 18 months. Mult Scler 17: 368-371, 2011.

17. Guzmán-Soto I, Salinas E, Hernández-Jasso I, Quintanar JL. Leuprolide acetate, a GnRH agonist, improves experimental autoimmune encephalomyelitis: a possible therapy for multiple sclerosis. Neurochem Res 37: 2190-2197, 2012.

The Internal Medicine is an Open Access journal distributed under the Creative Commons Attribution-NonCommercial-NoDerivatives 4.0 International License. To view the details of this license, please visit (https://creativecommons.org/licenses/ by-nc-nd/4.0/).

(C) 2020 The Japanese Society of Internal Medicine

Intern Med 59: 3093-3096, 2020 